\title{
Trois papyrus byzantins de Vienne
}

\section{Proposition de liturges}

\author{
P. Vindob. Gr. inv. 29966A et B Fig. 1 Hermopolite \\ $13,5 \times 15,5 \mathrm{~cm} ; 14,4 \times 14,1 \mathrm{~cm}$ \\ 22 juillet 362 après J.-C.
}

A. - Papyrus brun moyen ; encre brun foncé. Les bords gauche, droit et inférieur sont intacts. Le bord supérieur est cassé irrégulièrement. L'écriture est parallèle aux fibres et montre une main expérimentée, qui écrit avec une plume asscz grosse d'une façon cursive. Le papyrus a été plié au moins trois fois en hauteur. La marge au bas de la page est de $1,4 \mathrm{~cm}$. L'écriture ne continue pas tout à fait jusqu'au bord droit de la page. Sur le verso se trouvent quelques traces d'écriture, peut-être une adresse.

B. - Papyrus brun moyen; encre brun foncé. Le papyrus a été plié au moins quatre fois en hauteur et consiste actuellement pour une grande partie en fragments qu'on a assemblés lors de la restauration. Les bords gauche, droit et inférieur sont pour une grande partie restés intacts. Le bord supérieur est cassé irrégulièrement. La marge au bas de la page est de $2 \mathrm{~cm}$. L'écriture est parallèle aux fibres et continue jusqu'au bord droit de la page. Écriture cursive expérimentée. La deuxième main est la mème que celle qui a copié $29966 \mathrm{~A}$.

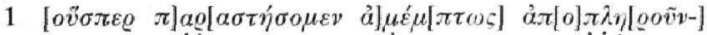

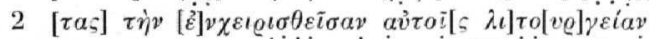

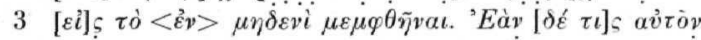

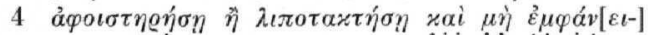

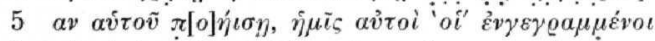

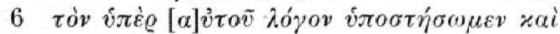

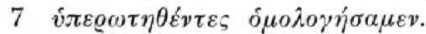

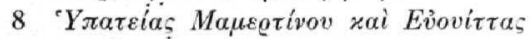

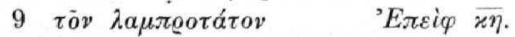

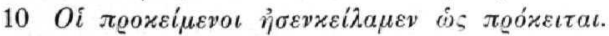

A

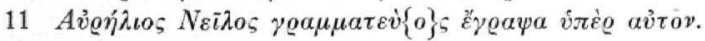

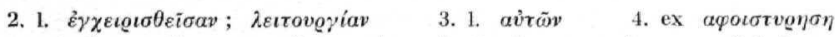

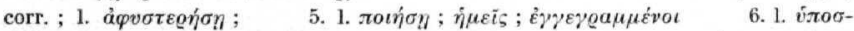

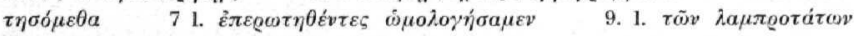
10 l. $\varepsilon \dot{i} \sigma \eta \gamma \gamma \varepsilon i \lambda a \mu \varepsilon v \quad 11$ l. $\alpha$ ข้น $\tilde{\omega} v$ 


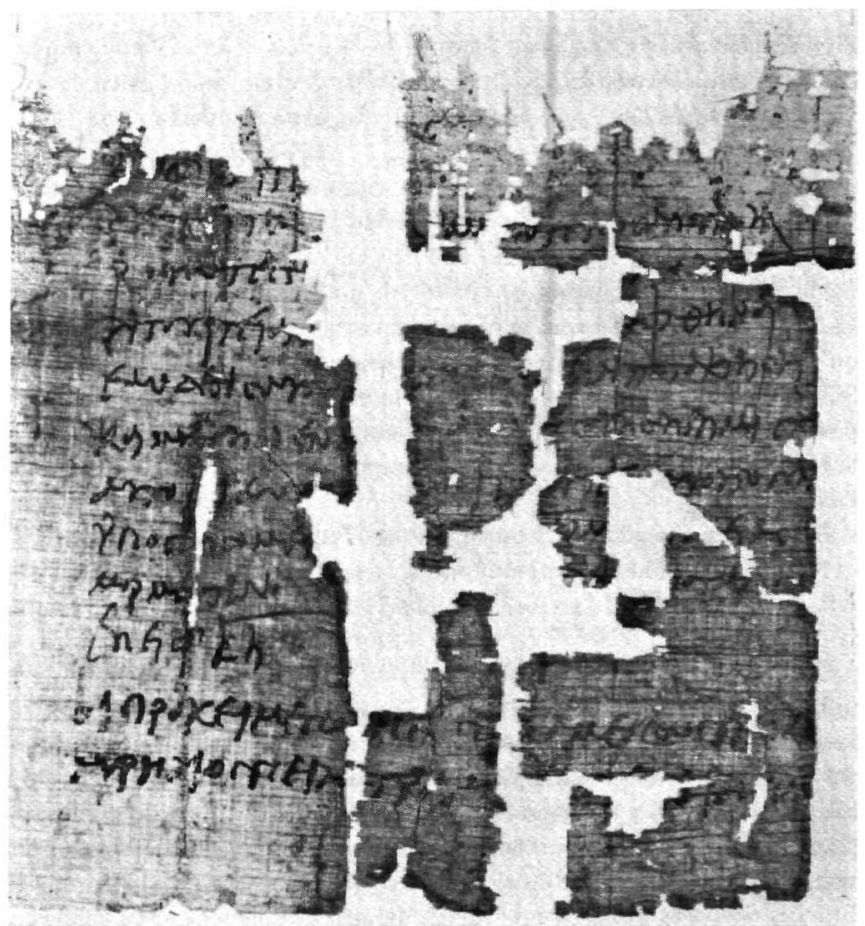

Fig. 1. - P. Vindob. Gr. Inv. 29966B (Hermopolite, 362).

B

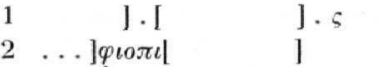

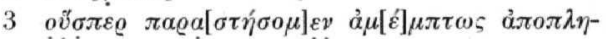

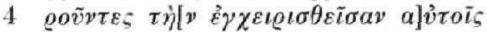

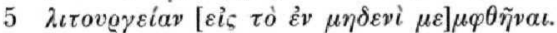

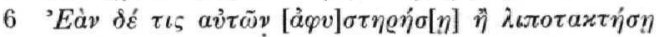

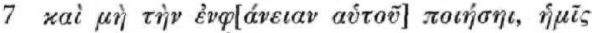

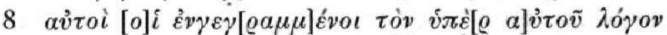

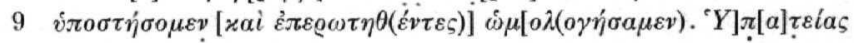




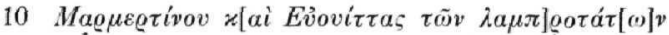

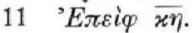

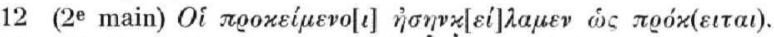

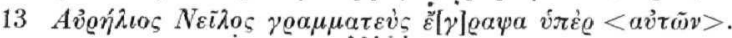

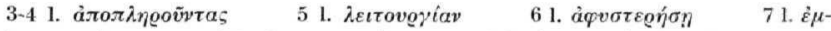

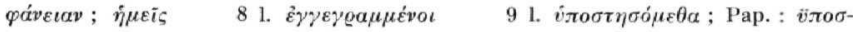

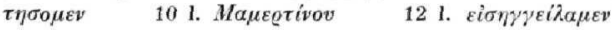

\section{Traduction :}

".... que nous mettrons à votre disposition, exécutant les liturgies qu'on leur imposera de façon impeccable à la satisfaction complète. Si, cependant, l'un d'entre eux tarde ou renonce à sa charge et ne fait pas acte de présence, nous, les personnes mentionnées ci-dessus, prendrons à notre charge la punition qui s'impose et après interrogation nous l'avons stipulé.

Pendant le consulat de Mamertinus et Evitta, le 28 Epeiph.

Nous, les personnes susmentionnées, (les) avons présentés comme décrit ci-dessus. Moi, le scribe, Aurelios Neilos, je l'ai écrit pour eux ".

La traduction de $29966 \mathrm{~B}$ est pratiquement pareille à celle de $29966 \mathrm{~A}$.

Les papyrus ci-dessus contiennent tous les deux la fin d'une présentation de liturges qui doivent ètre nommés. Tous les deux sont datés du 28 Epeiph du consulat de Mamertinus et Evitta, c'est à dire du 22 juillet 362 . Le caractère de la liturgie et le nombre de personnes proposées ne peuvent plus ètre précisés, parce que la partie du papyrus qui les concerne, est perdue. Seule la clause, où on garantit que les liturges proposés exécuteront leur tâche impeccablement, est conservée. Les personnes qui les présentent et dont on ne connait ni le nom ni la fonction, garantissent qu'ils sont prêts à subir eux-mêmes les sanctions imposées par l'État au cas oủ les liturges proposés feraient défaut.

Pour les différentes formes de déclarations de garantie dans le cadre d'attribution de liturgies, voir P. Vindob. Sijpesteijn 3 (=P.L. Bat. $\mathrm{XI}, 3$ ). Il distingue (pp. 17-18) deux catégories de documents, à savoir :

a) une déclaration dans laquelle quelques personnes proposent à un fonctionnaire de nommer une ou plusieurs personnes à la fonction de liturge. Dans ce cas, la partie qui fait la proposition garantit que les liturges exécuteront réellement leur liturgie. Voin $P$. Amh. II 139 ; P. Got. 5-6 ; P. Cairo Isid. 125 ; P. Lond. III 1246-1248 (p. 224-226). 
b) un document dans lequel on se porte garant pour un liturge déjà nommé que celuj-ci exécute sa liturgie, sous peine pour le garant de devoir prendre sa place (voir P. Lips. 45-51; P. Strassb. 46-51) ou de subir la sanction imposée (P. Herm. Rees 21; P. Vindob. Sijpesteijn 3-6, spéc. 3, note 1. 14-15).

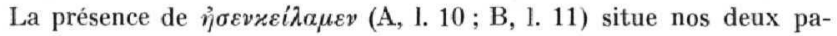
pyrus dans la première catégorie. Le fonctionnaire auquel les papyrus étaient adressés au départ est inconnu. Au cas où les liturges proposés étaient destinés à l'exécution de fonctions dans le conseil d'un village, il faudrait considérer le « Praepositus Pagi * comme la personne à laquelle on a adressé ce document (voir $P$. Leit. 3 , introd.).

On ne peut préciser si les deux documents concernent la même proposition de liturges. Il est cependant probable que les deux documents sont étroitement liés entre eux et il n'est pas exclu que $P$. Vindob. G 29966 A soit une copie de $P$. Vindob. G 29966B. Cette interprétation est corroborée par le fait que de telles propositions étaient souvent faites en plusieurs exemplaires (voir P. Lond. III 1246-1247; P. Cairo Preisigke 18-19).

Quant au fait que la souscription dans $P$. Vindob. G 29966B ne montre pas la mẻme écriture que le texte qui la précède, voir $P$. Cairo Isidor. $125,17-20$ : "Since the subscription is written by a second hand, this

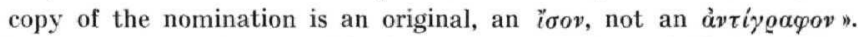

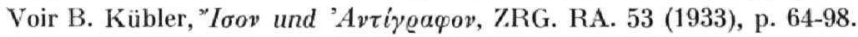
Comparer aussi P. Herm. Rees 55-56. A mon avis le scribe Aurelios Neilos a copié le document complet une deuxième fois. Ce qui est frappant, c'est qu'il a oublié, en $P$. Vindob. G inv. 29966B, 13, d'écrire $\alpha \dot{v} \tau \tilde{\omega} v$.

\section{Remarques :}

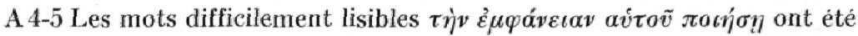

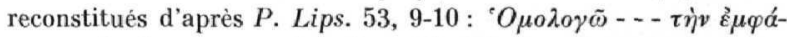

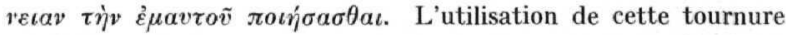
en discours direct ne se rencontre pas encore, à ce que je sache, dans les propositions de liturges ou de * Gestellungsburgschaften ».

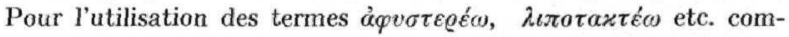
parer P. Vindob. Sijpesteijn 3, p. 20.

Pour la graphie de $\lambda \iota \pi о \tau \alpha \varkappa \tau \varepsilon ́ \omega$ voir P. Herm. Rees 21,17.

A $6{ }^{~ ' Y л о \sigma \tau \eta ́ б о \mu \varepsilon v ~ e ́ c r i t ~ a u ~ l i e u ~ d e ~ v л о \sigma \tau \eta \sigma o ́ ~} \mu \varepsilon \theta \alpha$ sous l'influence de

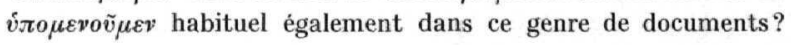


Les formes médiales des verbes gagnent d'ailleurs en influence par rapport aux formes actives. Voir S. G. Kapsomenakis, Voruntersuchungen..., p. $16 ; 130$.

A 7 Pour la stipulation, voir D. Simon, Studien zur Praxis der Sti-

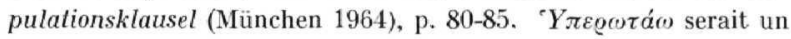
" addendum lexicis papyrologicis", mais, plus vraisemblablement, il s'agit ici d'une faute d'orthographe.

A 8 Pour les consuls Claudius Mamertinus et Flavius Nevitta, voir A. H. M. Jones - J. R. Martindale - J. Morris, Prosopography of the Later Roman Empire, Vol. 1, pp. 540-541 ; 626-627 ; PW.-RE XVII, Sp. 156 et suiv. Il est remarquable que le nom de Nevitta soit souvent défiguré dans les papyrus. Voir Preisigke WB III p. 75. Pour le génitif en - $\alpha_{\varsigma}$, comparer Ch. Döttling, Die Flexionsformen lateinischer Nomina in den griechischen Papyri und Inschriften, Diss. Bâle, Lausanne 1920, p. 29.

A 11 On trouve le nom Neilos avec une fréquence remarquable dans les papyrus de l'Hermopolite. On pourrait éventuellement en déduire l'argument que ces papyrus ont pour origine l'Hermopolite. De toute façon beaucoup de papyrus du rve siècle dans la collection viennoise proviennent de l'Hermopolite.

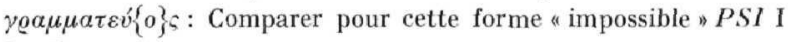
$86,7$.

\section{Relevé de paiements}

P. Vindob. Gr. inv. 14998

$13,2 \times 8,6 \mathrm{~cm}$
Fig. 2. Hermopolite

$1^{\mathrm{e}} \mathrm{m}$. du vve $^{\mathrm{e}}$ siècle après J.-C.

Papyrus brun clair ; encre noire. Le côté gauche est légèrement abîmé à la partie supérieure gauche; le côté supérieur est cassé irrégulièrement, le côté droit est cassé net sur un pli ou une collure, le côté inférieur est cassé mais sans perte d'écriture apparente. En bas de la page se trouve une marge de $1,8 \mathrm{~cm}$ environ. Du còté gauche, l'écriture commence immédiatement sans aucune marge; du côté droit, il y a une marge de $1,5 \mathrm{~cm}$ environ, dans laquelle se trouve le sigle des artabes. L'écriture, parallèle aux fibres, est une cursive du quatrième siècle ; cf. E. Boswinkel P. J. Sijpesteijn, Greek Papyri, pl. 36-38. 


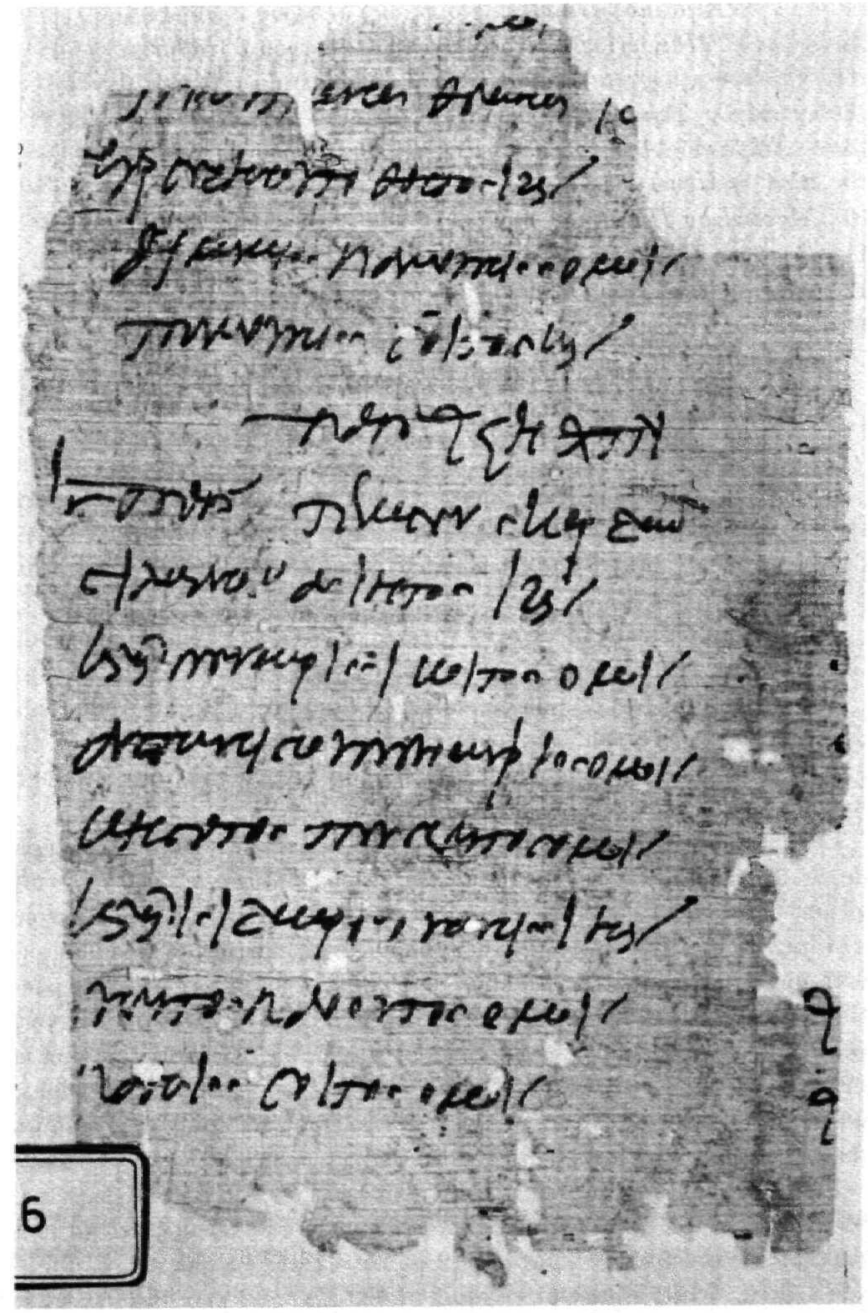

Fig. 2. - P. Vindob. Gr. Inv. 14996 (Hermopolite, 1e m. du rve siècle). 


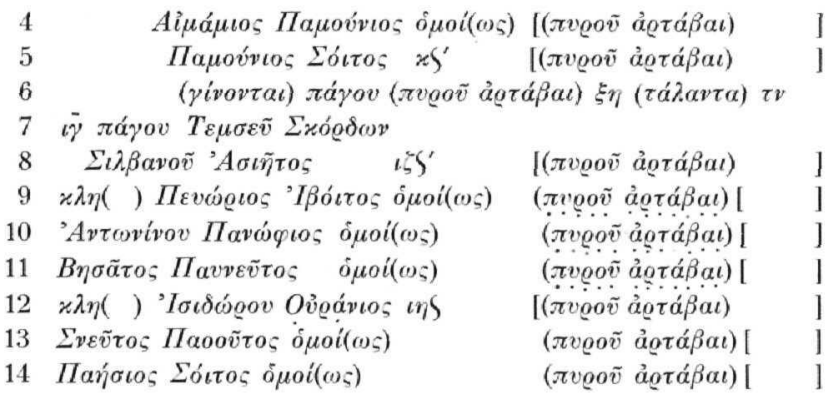

5 Pap. : бoïros 12 1. Ov̉eaviov

Le papyrus publié ci-dessus comporte une liste de noms de personnes. Derrière les noms, aux 11. 13-14, se trouve le sigle des artabes de blé. Ceci figure très probablement derrière chaque nom (voir les traces d'encre aux Il. 9-11).

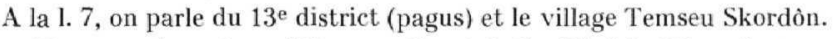
Ce village se situe dans l'Hermopolite (cf. P. Vindob. Worp 8, rem. l. 39). Ceci situe l'origine de ce papyrus. Comme connu l'introduction de la division des nomes en pagi est à dater en 307-308 après J.-C. (cf. P. Vindob. Worp 8, rem. 1. 6-7), un "terminus post quem " pour dater ce papyrus.

Les noms mentionnés dans ce papyrus sont en général bien connus de Fr. Preisigke NB et D. Foraboschi, Onomasticon alterum Papyrologicum. Seulement dans la 1.4 se trouve un nom nouveau, à savoir

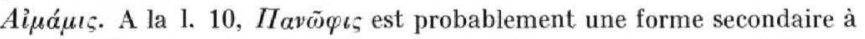
côté de Mavoṽqıs. Les 1. 6-7 nous font croire, que le papyrus fait partie d'un relevé, classé par pagi, des quantités de blé payées ou à payer, peut-être dans le cadre des impôts. A la 1. 6, on ne mentionne pas le numéro du pagus concerné. Comme à la 1.7 on parle du $13^{\mathrm{e}}$ pagus, on peut considérer qu'à la 1.6 on donne la quantité totale des artabes du $12^{\mathrm{e}}$ pagus.

La 1. 6 nous apprend qu'au moment où l'on écrivait ce papyrus, 68 artabes de blé représentaient une valeur de 340 talents, ce qui met l'ar tabe à 5 talents. Cf. pour le prix de blé à cette époque : A. C. Johnson - L. C. West, Byzantine Egypt, p. 176-177. Le prix fixé ici laisse supposer, en tenant compte du relevé de Johnson-West, l. c., que le 
papyrus doit dater du début de la première moitié du rve siècle après J.-C.

Les abréviations $x \lambda \eta \underline{o(--)}$, respectivement $x \lambda \eta(--)$, doivent être

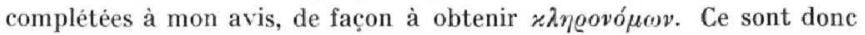
les héritiers qui paient. La résolution qui en ferait $x \lambda \hat{\eta} \varrho o v$ me paraît moins probable, parce que les impôts étaient en premier lieu payés par des individus ou leurs descendants.

\section{Quittance pour impôts perçus}

P. Vindob. Gr. jnv. 29967

HFRMOPOLITE

$6,4 \times 31,5 \mathrm{~cm}$.

vI ${ }^{e}$ siècle après J.-C.

Papyrus brun clair, encre noire. L'écriture, parallèle aux fibres, est une cursive expérimentée. La partie supérieure, du côté gauche, est légèrement abímée, mais sans perte de texte. Le papyrus étant plié au moins une fois en longueur, de petits trous sont visibles sur les plis. A gauche, il y a une marge de $1,5 \mathrm{~cm}$ environ. La marge supérieure est de $0,5 \mathrm{~cm}$ et celle du bas de 1 à $1,5 \mathrm{~cm}$. Du côté droit, l'écriture continue jusqu'au bord du papyrus.

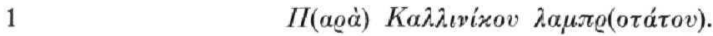

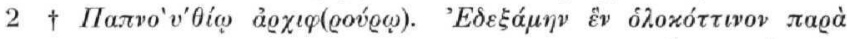

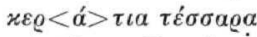

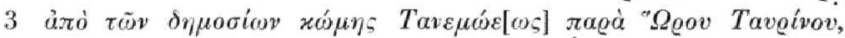
$\gamma i v(\varepsilon \tau \alpha \iota) \chi \varrho(v \sigma o \tilde{v}) \nu o(\mu \iota \sigma \mu \alpha \dot{\tau} \tau$ tov $) \alpha \pi(\alpha \varrho \alpha \dot{)}) \delta^{\prime \prime} \mu o^{\prime}(\alpha)$.

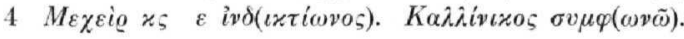

4 Pap. : iv $/$

\section{Traduction :}

«De la part du très brillant Kallinikos au chef des gardiens Papnouthios. J'ai reçu un solidus moins quatre keratia pour les impôts du village Tanemois de la part de Hôros, fils de Taurinos, soit un total de 1 nomismation d'or moins 4 (keratia) tel quel. Le 21 Mecheir de la 5 e indiction. Moi, Kallinikos, je marque mon accord. *

Ce genre de quittances se rencontre en grandes quantités dans $S P P$ III et VIII. Le Kallinikos, qui émet cette quittance est très probablement identique avec la personne du même nom dans SPP VIII 10491050 (cf. aussi SPP III 368-369; VIII 989). Notre texte peut, tout 
comme les textes mentionnés ci-dessus, être situé au vi ${ }^{\mathrm{e}}$ siècle après J.-C. La quittance concerne un montant d'1 solidus moins 4 keratia (cf. rem. l. 2) payé pour les impôts levés dans le village Tanemois.

\section{Remarques :}

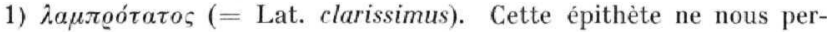
met pas de déterminer la position, que Kallinikos occupait dans la hiérarchie des fonctionnaires. Cf. P. Koch, Die byzantinischen Beamtentitel von 400-700, Diss. Jena 1903, p 20: "Schon zu Beginn der von mir behandelten Z.eit zeigt der Titel " clarissimus" die Neigung sich nach unten auszudehnen ». Cf. aussi O. Hornickel, Ehren- und Rangprädikate in den Papyrusurkunden, Diss. Giessen 1930, p. 22.

2) dexí@ovgos. Ce mot se trouve jusqu'ici uniquement dans P.S.I. VIII 938,5 ( $\mathrm{vI}^{\mathrm{e}}$ siècle après J.-C.; Aphrodito). Dans ce papyrus, on nous informe que ce fonctionnaire aura éventuellement mission de percevoir les dettes. Cf. aussi G. Rouillard, L'administration civile de l'Égypte byzantine, p. 47-48. Cf. P. J. Sijpesteijn, Chron. Ég. 48 (1973), pp. $121-126[=$ P. Amsterdam inv. 38, ligne 17].

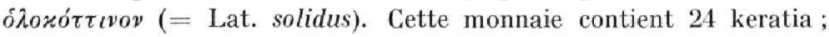
cf. L. C. West - A. C. Johnson, Currency in Roman and Byzanline Egypt, p. 137, s. v. $\chi \varrho v \sigma \iota x \alpha ́$. Comparer pour l'expression " $x$ nomismatia zagà y keratia ": J. Herrmann, Studien zur Bodenpacht, p. 112-113. Pour xegátıv, voir aussi H. L. Adelson, Leight Weight Solidi and Byzantine Trade during the Sixth and Seventh Centuries (New York 1957), p. 46 et suiv.

3) $\tau \dot{\alpha} \delta \eta \mu o ́ \sigma c \alpha$. Suivant Johnson-West (Byzantine Egypt, p. 259; 267), on indique par là souvent les impòts fonciers. Voir cependant aussi leur remarque (p. 305) : "In general $\delta \eta \mu o ́ \sigma \iota \alpha$ refers to taxes of any kind ». Cf. aussi Rouillard, o. c., p. 80 rem. 2 ; P. Mert. I $50,1$.

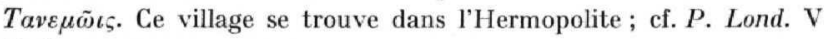
$1899,7$.

$\mu$ óva. Ceci indique qu'il n'y a pas de réduction du montant payé (cf. Johnson-West, o. c., p. 269 ; P. Princ. III 138, 5).

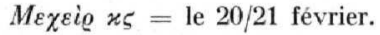

